\title{
One-day versus 3-day suprapubic catheterization after vaginal prolapse surgery: a prospective randomized trial
}

\author{
Annemarie Van Der Steen • Renee Detollenaere • \\ Jan Den Boon • Hugo Van Eijndhoven
}

Received: 18 June 2010 / Accepted: 4 January 2011 /Published online: 3 March 2011

(C) The Author(s) 2011. This article is published with open access at Springerlink.com

\begin{abstract}
Introduction and hypothesis For prolonged catheterization after vaginal prolapse surgery with anterior colporrhaphy, the optimal duration to prevent overdistention of the bladder remains unknown. We designed this study to determine the optimal length of catheterization.

Methods We conducted a prospective randomized trial in which 179 women were allocated to 1-day or 3-day suprapubic catheterization. The primary outcome was the duration of catheterization.

Results Mean duration of catheterization and hospital stay was significantly shorter in the 1-day catheterization group. The number of successful voiding trials was higher in the 3-day catheterization group $(90.9 \%$ versus $79.3 \%$ ), but this did not reach statistical significance. The percentage of urinary tract infection did not differ significantly between the groups (4.5\% versus $2.4 \%$ ). Conclusion Starting a voiding trial 1 day after vaginal prolapse surgery leads to shorter duration of catheterization and hospital stay.
\end{abstract}

Keywords Bladder retention - Catheterization · Urinary tract infection · Vaginal prolapse surgery

The study was conducted in the Department of Gynaecology of the Isala Klinieken, Zwolle, The Netherlands.

A. Van Der Steen · R. Detollenaere · J. Den Boon •

H. Van Eijndhoven

Isala Klinieken,

Zwolle, The Netherlands

\section{A. Van Der Steen $(\bowtie)$}

Department of Obstetrics and Gynaecology,

University Medical Center Groningen,

PO Box 30.001, 9700 RB, Groningen, The Netherlands

e-mail: a.vdsteen@yahoo.com

\section{Introduction}

After vaginal prolapse surgery with anterior colporraphy, it is common to use an indwelling catheter for several days in order to prevent overdistention of the bladder. In literature, duration of catheterization varies from $3 \mathrm{~h}$ to 7 days [1-3]. The benefit of short-term catheterization could be a reduction of hospital stay and urinary tract infections. The latter is important because urinary tract infections (or asymptomatic bacteriuria) account for $40 \%$ of the hospital-acquired infections and $80 \%$ of these infections are associated with the use of urinary catheters. On the other hand, a short period of catheterization is associated with more postoperative voiding problems, like obstructive voiding and large residual volumes, which could lead to bladder dysfunction and urinary tract infection [3, 4]. Therefore, good timing of catheter removal is very important to reduce postoperative morbidity. In spite of the widespread use of postoperative catheterization, welldesigned studies on this subject are sparse. In one randomized controlled trial comparing 1-day to 5-day postoperative transurethral catheterization, a tenfold decrease of urinary tract infections was demonstrated. However, in the short-term catheterization group, $40 \%$ of the patients had large post voiding residuals with the necessity of recatheterization. It was suggested that the optimal timing of catheter removal is sometime between 1 and 5 days [5]. A trial performed by Schiøtz compared 1-day catheterization with 3-day catheterization and showed that the catheter can safely be removed on the first postoperative day [6].

We designed a prospective randomized trial in which the 3-day suprapubic catheterization protocol used in our hospital was compared with 1-day suprapubic catheterization. We hypothesized that 1-day catheterization leads to a shorter 
duration of catheterization and reduction of urinary tract infections.

\section{Materials and methods}

The study protocol was approved by the local medical ethical committee. Between October 2007 and February 2009 , all patients undergoing vaginal prolapse surgery with anterior colporrhaphy were asked for an informed consent for this study. Exclusion criteria were preoperative need for catheterization, contraindication for suprapubic catheterization, inability to give an informed consent, or preoperative urinary tract infection.

Before prolapse surgery, a urinary sample was taken for urinalysis for the detection of urinary tract infection. All patients received preoperatively $1,000 \mathrm{mg}$ cefazolin and $500 \mathrm{mg}$ metronidazole as antibiotic prophylaxis. Anterior colporrhaphy was performed according to the standard plicating technique. In general, no Kelly sutures were used. At the end of surgery, a suprapubic catheter was inserted (Cystofix ${ }^{\circledR}$, Braun). The use of a suprapubic catheter was the standard in our hospital at the time of the study partly because it is more comfortable for the patient during the postoperative period, it is easier when prolonged catheterization is necessary, and patients can continue performing their voiding trials at home.

Patients were randomized into a 1-day or 3-day catheterization group using sealed opaque envelopes. A variable block size was used for the randomization.

On the first or third morning after surgery, the catheter was clamped and patients were encouraged to void as soon as possible. When this occurred within $4 \mathrm{~h}$, the post voiding residual volume was measured by taking the clamp off the catheter. If the patient was unable to void within $4 \mathrm{~h}$, the residual volume was also measured, and the catheter was clamped again for a period of $4 \mathrm{~h}$. If the patient was unable to void during the day, the catheter was opened overnight and clamped again the following morning. In case of residual volumes of more than $100 \mathrm{ml}$, the training was continued; overnight the catheter was opened and clamped again the next day. A successful voiding trial was defined as a residual volume of less than $100 \mathrm{ml}$ twice in a row within $48 \mathrm{~h}$ after clamping the catheter. Hereafter, a urinary sample was taken from the catheter for sedimentation and culture and the catheter was removed. A urinary tract infection was diagnosed in case of a positive culture $\left(10^{5}\right.$ of one type of bacteria) in combination with fever, dysuria, or leucocytes in the sedimentation. If the suprapubic catheter had to be removed before or during the voiding trial (leakage or clotting), a transurethral catheter was placed if necessary.
The Foley catheter was removed according to the allocation of the patient on the first or third postoperative day and the residuals were measured using a bladder scan.

The primary outcome measure was defined as the mean duration of catheterization from the day of surgery until the suprapubic catheter was removed. Secondary outcome measures were successful removal of the catheter within 2 days after the start of voiding trial, urinary tract infections, hospital stay, and residual volumes.

\section{Statistics}

With the standard protocol used in our hospital, mean duration of catheterization was 4.6 days. With a decrease of this primary outcome measure of 2 days, a sample size of 82 persons per group was calculated for $80 \%$ power and $5 \%$ type 1 error probability. As per protocol analysis was performed and as the variables had a normal distribution, a Student's $t$ test was used. For comparing the nominal results, a Fisher's exact test was used.

\section{Results}

A total of 269 patients were planned for an anterior colporrhaphy and were informed about the study. Of this group, 219 gave informed consent. Of these 219 patients, 40 were excluded after surgery because they did not meet the criteria for randomization (Fig. 1). This was mainly because the planned anterior colporrhaphy was not performed or the suprapubic catheter could not be inserted. After surgery, 179 patients were randomized, 87 in the 1-day catheterization group and 92 in the 3-day catheterization group. Of these groups, 82 and 88 patients respectively were analyzed. In five patients, the suprapubic catheter did not function properly (clotting or leakage) and was replaced by a transurethral catheter during the voiding trial. Nine patients were not analyzed because of violation of the protocol. These patients did not start voiding trial on the first or third morning according to their randomization.

The baseline characteristics were similar in both groups regarding type of surgery and age, with a mean age of 60 in both groups (Table 1). Time of surgery was equal in both groups. Both mean duration of catheterization (2.13 versus 3.55 days) and hospital stay (2.94 versus 3.46 days) were significantly shorter in the group with the catheter clamped the first postoperative day (Table 2). Although clamping of the catheter on the first day after surgery resulted in a lower number of successful trials of voiding, $79.3 \%$ versus $90.9 \%$, this did not reach statistical significance. Shorter duration of catheterization did not result 
Fig. 1 Flowchart of randomization. The main reason for exclusion of the study was because the planned anterior colporrhaphy was not performed or the suprapubic catheter could not be inserted. Drop out was due to protocol violation

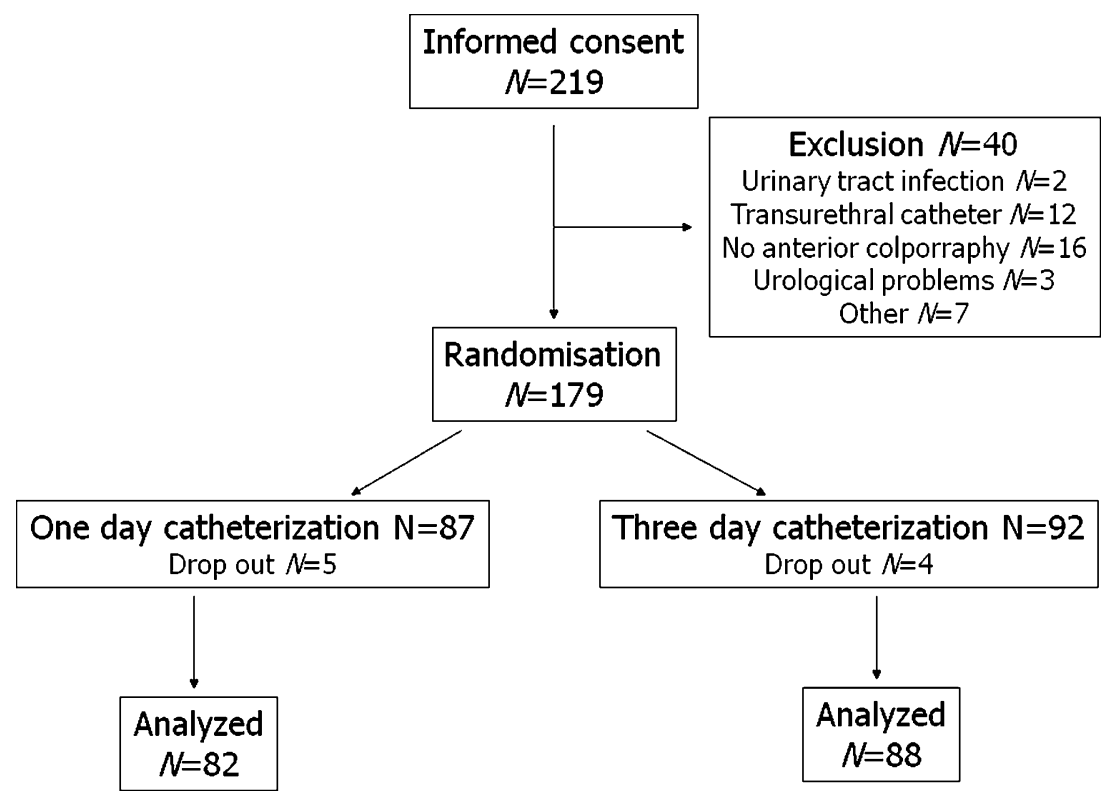

in a lower number of urinary tract infections, $2.4 \%$ versus $4.5 \%$ (Table 2). However, we were not able to obtain the urinary sample in $1.2 \%$ of patients in the 1-day group and in $25 \%$ of patients of the 3 -day group. The number of patients going home with the catheter in place was equal in both groups (Table 2). The median first post void residual volumes were significantly higher in the 1-day catheterization group, 50 versus $20 \mathrm{ml}(p=0.016)$.

\section{Discussion}

Although prolonged catheterization after prolapse surgery is believed to prevent voiding problems, little is known about the optimal timing of catheter removal. Our data demonstrate that clamping of the suprapubic catheter and starting voiding trials on the first postoperative day results in a shorter duration of catheterization and hospital stay, without a difference in urinary tract infections and long-term catheterization.

Some bias in the results cannot be excluded because the nurses on the ward conducting the catheterization process were not blinded for allocation. Expectations of the nurses of the outcome on one hand and the fact that they were used to the 3-day catheterization protocol on the other hand could have influenced the results. We believe that after they were convinced that a voiding trial on the first postoperative day can be successful, they were better able to encourage their patients to mobilize and void on the first day. This was confirmed by the fact that after the nurses were used to the study protocol, the results of the 1-day catheterization group improved.

Table 1 Baseline characteristics

Age at surgery

Manchester-Fothergill
Manchester-Fothergill + transobturator tape
Prolift + anterior colporrhaphy
Prolift + anterior colporrhaphy + transobturator tape
Sacrospinous fixation + anterior colporrhaphy
Sacrospinous fixation + anterior colporrhaphy +
transobturator tape
Vaginal hysterectomy + anterior colporrhaphy
Anterior colporrhaphy
Anterior colporrhaphy transobturator tape

One-day catheterization Three-day catheterization $(n=82)$

60 $(n=88)$

$\begin{array}{rr}n & n \\ 3 & 5 \\ 0 & 1 \\ 1 & 3 \\ 1 & 0 \\ 44 & 44 \\ 3 & 3 \\ 21 & 24 \\ 8 & 6 \\ 1 & 2\end{array}$


Table 2 Results

\begin{tabular}{|c|c|c|c|c|c|c|}
\hline & & One-d & ization & Three- & rization & \\
\hline & & Mean & $\pm \mathrm{SD}$ & Mean & $\pm \mathrm{SD}$ & $p^{\mathrm{a}}$ \\
\hline & $\begin{array}{l}\text { Duration of } \\
\text { catheterization (days) }\end{array}$ & 2.13 & \pm 1.66 & 3.55 & \pm 2.11 & 0.000 \\
\hline & $\begin{array}{l}\text { Duration of hospital } \\
\text { stay (days) }\end{array}$ & 2.94 & \pm 1.00 & 3.46 & \pm 0.78 & 0.000 \\
\hline & & $n$ & $\%$ & $n$ & $\%$ & $p^{\mathrm{b}}$ \\
\hline & Successful voiding trial & $65 / 82$ & 79.3 & $80 / 88$ & 90.9 & 0.063 \\
\hline & $\begin{array}{l}\text { Discharge from hospital } \\
\text { with catheter }\end{array}$ & $3 / 82$ & 3.7 & $3 / 88$ & 3.4 & 0.627 \\
\hline Student's $t$ test & Urinary tract infection & $2 / 82$ & 2.4 & $4 / 88$ & 4.5 & 0.683 \\
\hline
\end{tabular}

The low first post voiding residual volumes in both groups were probably another side effect of the study. Because the following residual volumes in general were much higher, one can assume that the first attempt to void was made very shortly after clamping the suprapubic catheter with only a low urinary bladder volume. Therefore, we believe that the difference in first post void residual between the two groups is not clinically important.

Finally, the comparison of the standard protocol with a new protocol also led to the problem that we were very aware of taking the urinary sample in the 1-day group, but that this was not always done in the 3-day group, $1.2 \%$ versus $25 \%$ of data was missing. Also the nurses might have been more likely to take a urinary sample if they expected a urinary tract infection in the patient. This raises the possibility that there were other protocol violations; however, no other violations were noticed. Nevertheless, we found a similar small number of urinary tract infections in the two groups, $2.4 \%$ versus $4.5 \%$. As all patients received prophylactic antibiotics, this could not have influenced a difference in urinary tract infections between the groups. Moreover, the percentage of urinary tract infections in the two groups is very small and in our opinion it is not clinically significant.

To our knowledge only three prospective studies have been elaborated comparing prolonged catheterization to 1-day catheterization $[2,5,6]$. In the Dutch prospective study of Hakvoort et al., 1-day catheterization was compared to 5 days, which today might be considered as an uncommon long period of catheterization. The data of this study were comparable to our results. Percentage of successful trial of voiding was lower in the 1-day catheterization group (60\%), probably because of the use of a transurethral catheter. That is to say, with high post voiding residuals the replacement of an indwelling catheter was necessary, while in our protocol trial of voiding was continued [5].

Schiøtz also performed a randomized controlled trial comparing 1-day versus 3-day postoperative catheterization after vaginal prolapse surgery. In this study, 165 women were randomized to 1-day or 3-day catheterization with a transurethral Foley catheter. Although no difference in postoperative urinary retention was found, the need for recatheterization in the 1-day group was higher. No significant difference in urinary tract infections was demonstrated between the groups [6].

Glavind et al. compared 3-h catheterization to 24-h catheterization after vaginal prolapse surgery. They examined 140 women allocated to removal of the vaginal pack and urinary catheter $3 \mathrm{~h}$ after surgery or the next day. Postoperative bleeding was equal in both groups, as was the need of intermittent catheterization, $1 \%$ versus $5 \%$, which were very low percentages compared to other studies. However, post voiding residuals were not measured in this study. They concluded that early postoperative removal of the vaginal pack and catheter did not result in more postoperative bleeding or voiding difficulties, and could possibly avoid risks of urinary tract infection [2].

Although postoperative removal of the catheter a few hours after surgery could have benefits, the effectiveness of this approach in case of more extensive prolapse surgery remains questionable. In our study, the majority of patients who failed the voiding trial on the first day had concomitant surgery such as a vaginal hysterectomy or sacrospinous fixation with anterior repair. We believe the inability to void was at least partly caused by immobilization of the patient after this type of surgery. If surgery is limited to an anterior colporrhaphy, voiding trials probably can start on the day of surgery. Unfortunately, our numbers were too small to reliably analyze these subgroups. More research is needed to optimize and individualize the duration of postoperative catheterization based on factors such as extent of surgery and age.

Our data reveal that postoperative catheterization after vaginal prolapse surgery could be limited to 1-day catheterization. Prolonged catheterization of more than 1 day leads to significantly longer hospitalization. To this moment, it is unclear what the best treatment is for patients with high post voiding residual volumes more than 2 days after surgery. The latter question will hopefully be answered by an ongoing Dutch study in which clean intermittent catheterization is 
compared to indwelling catheterization in this group of patients (www.trialregister.nl NTR1152).

Authors' contributions AS designed and performed the trial and was responsible for drafting the manuscript. $\mathrm{RD}$ and JB helped performing the trial; HE had the overall responsibility for the study and manuscript.

\section{Conflicts of interest None.}

Open Access This article is distributed under the terms of the Creative Commons Attribution Noncommercial License which permits any noncommercial use, distribution, and reproduction in any medium, provided the original author(s) and source are credited.

\section{References}

1. Hakvoort RA, Burger MP, Emanuel MH, Roovers JP (2009) A nationwide survey to measure practice variation of catheterisation management in patients undergoing vaginal prolapse surgery. Int Urogynecol J 20:813-818

2. Glavind K, Mørup L, Madsen H, Glavind J (2007) A prospective, randomised, controlled trial comparing 3 hour and 24 hour postoperative removal of bladder catheter and vaginal pack following vaginal prolapse surgery. Acta Obstet Gynecol Scand $86: 1122-1125$

3. Ottesen M, Sørensen M, Rasmussen Y, Smidt-Jensen S, Kehlet H, Ottesen B (2002) Fast track vaginal surgery. Acta Obstet Gynecol Scand 81:138-146

4. Niël-Weise BS, van den Broek PJ (2005) Urinary catheter policies for short-term bladder drainage in adults. Cochrane Database Syst Rev. doi:10.1002/14651858.CD004203.pub2

5. Hakvoort RA, Elberink R, Vollebregt A, Van der Ploeg T, Emanuel MH (2004) How long should bladder catheterisation be continued after vaginal prolapse surgery? a randomised controlled trial comparing short term versus long term catheterisation after vaginal prolapse surgery. BJOG 111:828830

6. Schiøtz HA (1995) Comparison of 1 and 3 days' transurethral Foley catheterization after vaginal plastic surgery. Int Urogynecol J $6: 158-161$ 